\title{
BMJ Open Defining compassion in the digital health age: protocol for a scoping review
}

\author{
David Wiljer, ${ }^{1,2,3,4,5}$ Rebecca Charow, ${ }^{1}$ Helen Costin, ${ }^{4,6}$ Lydia Sequeira, ${ }^{2,5}$ \\ Melanie Anderson, ${ }^{7}$ Gillian Strudwick, ${ }^{2,5}$ Tim Tripp, ${ }^{7}$ Allison Crawford ${ }^{3,5,6}$
}

To cite: Wiljer D, Charow $\mathrm{R}$, Costin $\mathrm{H}$, et al. Defining compassion in the digital health age: protocol for a scoping review. BMJ Open 2019;9:e026338. doi:10.1136/ bmjopen-2018-026338

- Prepublication history for this paper is available online To view these files, please visit the journal online (http://dx.doi. org/10.1136/bmjopen-2018026338).

Received 27 August 2018 Revised 11 December 2018 Accepted 20 December 2018

Check for updates

(c) Author(s) (or their employer(s)) 2019. Re-use permitted under CC BY-NC. No commercial re-use. See rights and permissions. Published by BMJ.

1 UHN Digital, University Health Network, Toronto, Ontario,

Canada

${ }^{2}$ Institute of Health Policy Management and Evaluation, University of Toronto, Toronto, Ontario, Canada

${ }^{3}$ Department of Psychiatry, University of Toronto, Toronto, Ontario, Canada

${ }^{4}$ The Wilson Centre, University Health Network, Toronto,

Ontario, Canada

${ }^{5}$ Centre for Addiction and Mental Health, Toronto, Ontario, Canada

${ }^{6}$ Medical Psychiatry Alliance, University of Toronto, Toronto, Ontario, Canada

${ }^{7}$ Library and Information

Services, University Health

Network, Toronto, Ontario, Canada

Correspondence to

Dr David Wiljer;

david.wiljer@uhn.ca

\begin{abstract}
Introduction The notion of compassion and compassionate care is playing an increasingly important role in health professional education and in the delivery of high-quality healthcare. Digital contexts, however, are not considered in the conceptualisation of compassionate care, nor is there guidance on how compassionate care is to be exercised while using digital health technologies. The widespread diffusion of digital health technologies provides new contexts for compassionate care, with both opportunities for new forms and instantiations of compassion as well as new challenges. How compassion is both understood and enacted within this evolving, digital realm has not been synthesised.
\end{abstract}

Methods and analysis This scoping review protocol follows Arksey and 0'Malley's methodology to examine dimensions of compassionate professional practice when digital technologies are integrated into clinical care. Relevant peer-reviewed literature will be identified using a search strategy developed by medical librarians, which applies to six databases of medical, computer and information systems disciplines. Eligibility of articles will be determined using the two-stage screening process consisting of (1) title and abstract scan, and (2) fulltext review. Screening, abstracting and charting will be conducted by two independent reviewers, with a third reviewer available for resolution when consensus is not achieved. In order to look at the range of current research in this area, extracted data will be thematically analysed and validated by content experts. Descriptive statistics will be calculated where necessary.

Ethics and dissemination Research ethics approval and consent to participate is not required for this scoping review. The results of the review will inform resource development and strategy for Associated Medical Services (AMS) Healthcare, a Canadian charitable organisation at the forefront of advancing research and leadership development in health and humanities, as part of the AMS Phoenix Project: A Call to Caring, particularly for digital professionalism frameworks so that they are inclusive of a compassion competency.

\section{INTRODUCTION}

Compassion is a topic of great interest in recent healthcare research. ${ }^{1}$ Scholars, health professionals and patients across healthcare disciplines have agreed that compassion in health services is a necessary and critical component of healing. ${ }^{2-4}$ This value is reflected widely.
Strengths and limitations of this study

Findings from this review will contribute to the development of a definition of compassionate care within the context of digital health.

- The methodology includes a transparent and rigorous study design grounded in established, evidence-based frameworks (Arksey and 0'Malley), a systematic search strategy developed collaboratively and iteratively with a medical librarian, and standardised charting using a theory-oriented framework.

- Our team is composed of health services researchers from both the compassionate care and digital health fields, including senior investigators who are content experts.

- No quality assessments will be completed on the included studies since our objective is to provide a baseline understanding of the state of compassion in the field of digital healthcare innovations.

- Findings will be limited to articles written in English, and thus subject to cultural bias.

An imperative of the Canadian Medical Association, for example, specifies that our care system must 'optimise function and compassionate relief of suffering,. ${ }^{5}$ Compassionate practice is similarly reflected in paradigms of health education. As health education shifts towards competency-based frameworks globally, the quality and practice of compassion become milestones of professionalism and one's role as a 'communicator', as outlined by the American Association of Medical Colleges, ${ }^{6}$ Accreditation Council for Graduate Medical Education, ${ }^{7}$ CanMEDS framework $^{8}$ and others. ${ }^{9}$

Despite the prevalence of compassion in the current discourse, there is a growing disparity between what we know about the healing benefits of compassion in health services and the feedback from service users and providers about the lack of compassion in the current health system. There have been numerous conceptualisations of compassion across healthcare disciplines including nursing, social work, psychology 
and medicine,${ }^{10-12}$ yet commonalities exist across definitions, including (1) an initial recognition of suffering by an individual followed by either (2) an emotional or (3) operative response to this suffering. ${ }^{12}$ For example, the Oxford Handbook of Compassion Science proposes that compassion is a 'discreet and evolved emotional experience' that an individual has on recognising and wanting to alleviate the suffering or perceived unmet needs of another. ${ }^{13}$ This working definition of compassion involves several distinct components including the (1) awareness of another's experience of suffering or need, (2) feeling 'moved' and (3) recognising this feeling as a response to the other's need; (4) making a judgement that the other is suffering; and (5) engaging in a behaviour in an attempt to alleviate the suffering. ${ }^{13}$ Some scholars have suggested that the shift towards efficiency in healthcare service poses limitations to the time that compassionate practice requires. ${ }^{14} 15$

Digital technologies, especially modalities for communication, are one of the sets of tools increasingly leveraged to facilitate this shift towards efficiency and accessibility. ${ }^{16}$ Within health service improvement, this intersection of technology and healthcare gave rise to the discipline of 'eHealth'. ${ }^{17}$ A widely used definition of eHealth states that this is "an emerging field in the intersection of medical informatics, public health and business, referring to health services and information delivered or enhanced through the Internet and related technologies". ${ }^{18}$ Over time, eHealth evolved to encompass all digital modalities involved within healthcare, including but not limited to telemedicine, electronic medical records and mobile health, leading to the larger umbrella term of "digital health'. Digital health, which refers to the 'use of information technology and electronic communication tools within the delivery of healthcare services', has accelerated its adoption over the past two decades. ${ }^{19}$ Through implementing electronic health records and using mobile applications, digital health has led to improved quality, safety and efficiency in healthcare. ${ }^{20}$ These technologies have propelled all areas of health communication, including knowledge transfer between patients and health professionals, education, decision support and health promotion. ${ }^{21}$ For some patients, the increased anonymity and distance can support better empathic and honest communication than in-person consults, especially within online healthcare communities. ${ }^{22}$ Digital health has also improved accessibility of health services and the skills of health professionals to rural and remote communities and those patients with limited mobility. ${ }^{23}$ However, although there may be potential benefits, digital health represents a transformational shift in the context, process and manner that care is delivered. With the transitions in care including mobile computerised nursing stations, touch screens, various automated processes and services, and barcode medication administration, a new mediating entity emerges in the traditional patient-provider relationship and compassionate care-technology. ${ }^{24} 25$ This technology can be as simple as a user interface for automatic self-registration or an artificial intelligence robot that contributes to bedside care.

As we move beyond simple automation of healthcare processes, health technologies will increase the potential for patient engagement and activation, and change the nature of the therapeutic relationship. Through mobile apps, patients have the opportunity to become more effective partners in their care, with increased access to their data and ability to track their symptoms more accurately. ${ }^{26}$ Through web-based platforms, patients are electing to engage in computer-based communication with their providers ${ }^{27}$ as well as psychological interventions. ${ }^{28} 29$ This widespread diffusion of digital health provokes a new discourse on information management, dissemination and control. ${ }^{30}$ With this increased patient engagement, the boundaries of professional ethics and expectations are changing as the capabilities for real-time two-way communication between health professionals and patients are now available through email, texting, in-application chats and video-calling. ${ }^{31}$ Through the removal of geographical and temporal barriers, information and resources are available anytime, anywhere. This increase of availability can potentially lead to an increased workload and information overload by health professionals, who have to grapple with knowing when to 'turn off' ${ }^{32}$ Moreover, communication and care through digital means could lack emotional signals and cues to convey empathy that may be present in the traditional, in-person encounter. This has led to a call for integrating digital empathy into the curriculum of health professionals. ${ }^{33}$ Shifting medical practice requires new competencies to ensure that providers are able to sustain a patient-centred approach through creatively and flexibly using digital health applications while maintaining attention to the patient's narrative. ${ }^{33}$ Improved communication skills, self-reflection and reflexivity are examples of focused competencies to help professionals examine their interactions and express empathy and compassion in a digital world. ${ }^{34}$

In recent publications, the Associated Medical Services (AMS) suggested that education for healthcare students integrate the use of new technologies into practice, as well as promote compassionate care and the humane aspects of care. ${ }^{35}{ }^{36}$ Although work is underway to define what competencies are necessary for the practice of care within digital health contexts, compassion as a distinct competency itself has yet to be considered in the digital realm. ${ }^{37}$ Furthermore, frameworks for digital professionalism ${ }^{39}$ presently lack consideration of or guidance on how digital health technologies are to be incorporated into the practice of compassionate care, and digital contexts are often not considered in the conceptualisation of compassionate care. This divide between the discourse of digital and the discourse of compassion in healthcare is illustrated by the fact that digital health technologies are not explicitly mentioned in the Handbook of Compassion Science's table of contents. ${ }^{13}$ Could it be that there is a paradox inherent in the concept of digital 
compassion? Perhaps there exists a latent assumption that compassion requires an intimate, human-to-human interaction? Might compassion, therefore, be considered unachievable via and incompatible with the developing technological modalities in healthcare systems? To these authors' knowledge, how compassion is both understood and enacted within the digital realm remains unexplored terrain.

Moving forward, we need a better understanding of the intersection of compassionate care in digital contexts through considering the barriers and facilitators of technology, as well as technology's intended and unintended consequences on care delivery and healthcare interactions. Based on changing health delivery contexts and modalities, we need to identify the competencies and skills that are required for practising compassionately. This review is intended to contribute to the conversation and aid health professionals and educators in practising and teaching compassionate digital care, with the hopes to even inform software design in the future. Ensuring that compassion does not get lost within digital health is important at the level of the clinical encounter and also for the health system in whole so that we can respond to the rapidly evolving needs of patients and providers.

In reviewing the digital and compassion literature, we plan to discuss this concept from the following three perspectives: (1) from a patient-provider perspective, unique changes in styles of communication and information exchange in the context of digital care; (2) from a professional perspective, changes in required education, ethical conduct, scope of practice and practice guidelines; (3) from a systems perspective, changes in organisational policies and health professional workflows adapted to support compassionate digital healthcare.

\section{METHODS}

This protocol outlines a review that aims to define what it means to provide compassionate care in the digital sphere, that is, 'digital compassion'. We seek to inform this definition by examining the evidence-based literature on healthcare technologies. We will outline potential factors that facilitate and/or hinder compassion when health technologies are integrated into health professional workflows and patient experiences. The findings from this review will be used by AMS Healthcare whose current priority, The AMS Phoenix Project, is on compassionate care through curriculum development, adoption of standards, as well as building and supporting a community of clinical change leaders with publicly available resources. ${ }^{40}$ Furthermore, we believe this general knowledge synthesis is a critical first step to developing conceptual and definitional frameworks for the future success of educational interventions.

This topic has not yet been addressed in the literature, to the study team's knowledge. Given this knowledge gap, we have chosen to follow Arksey and O'Malley's methodological framework for scoping reviews to allow for broad exploration of how compassion is discussed in the digital literature as a precursor for future systematic reviews. ${ }^{41-43}$ While this is not a systematic review, this protocol will also follow relevant aspects of the Preferred Reporting Items for Systematic Review and Meta-Analysis Protocols guidelines to ensure transparency and rigour in reporting the methodology. ${ }^{445}$

To establish a baseline understanding about how compassion is operationalised when using digital technologies reported in both academic and grey literature, this review will answer the following questions:

1. How is compassion discussed in the digital health literature?

2. What are the factors that facilitate or hinder compassion as reported in the digital health literature?

3. What professional practice issues are addressed in this literature, including educational and clinical adaptations, training, pedagogical and clinical methods for providing compassionate care in a digital environment?

\section{Search strategy}

The search strategy was iteratively developed with experienced medical librarians (MA, TT) at the Toronto General Hospital in Toronto, Canada. The search strategy was first developed for Medline (table 1; table 1 legend) and then translated to query five other databases in March 2018. Since the research that is relevant to our topic is likely to be found in fields outside of medicine, we chose to search the following databases: Web of Science; ProQuest Computer Science and Computer and Information Systems Abstracts; Medline; PsycINFO; Embase; CINAHL (Cumulative Index of Nursing and Allied Health Literature). This range of databases will capture a comprehensive sample from the following disciplines: health sciences, psychiatry/psychology, social work, nursing, allied health, and computer and information systems sciences. If the search results include conference abstracts and proceedings, a subsequent search to find any corresponding follow-up articles will be conducted in Google Scholar. Finally, a 'pearl growing' also known as 'handcomb' process will also be conducted where all cited works in the included articles from the initial screening will also undergo the two-stage screening.

Both 'compassion' and 'digital' include a range of concepts/technologies and are defined in different ways in the literature of the fields in which we are interested. As a result, we are searching using index terms (subject headings) and appropriate keywords for three topics:

1. Computers, internet, smart phones, programs, applications, informatics, electronic records, e-health, telemedicine and so on AND

2. Compassion, empathy, sympathy, emotional intelligence, humanism, and so on AND

3. Communication or relationships between doctors and patients, person-centred, patient-centred, therapeutic alliance and so on.

The results from the search will be exported into the citation manager EndNote (http://endnote.com/), 


\begin{tabular}{|c|c|c|}
\hline$\#$ & Searches & Results \\
\hline 1 & exp Medical Informatics/ & 418356 \\
\hline 2 & (digital?? or e-health or ehealth).mp. & 132134 \\
\hline 3 & exp Computer Systems/ & 162400 \\
\hline 4 & (info* adj4 tech*).mp. & 26209 \\
\hline 5 & computer*.mp. & 734409 \\
\hline 6 & (health* adj4 tech*).mp. & 21559 \\
\hline 7 & online.mp. & 85504 \\
\hline 8 & (e-portal* or eportal*).mp. & 15 \\
\hline 9 & $\left(\right.$ patient $^{*}$ adj2 portal*).mp. & 4046 \\
\hline 10 & $\begin{array}{l}\text { exp medical records systems, } \\
\text { computerized/ }\end{array}$ & 34754 \\
\hline
\end{tabular}

\begin{tabular}{|c|c|c|}
\hline 11 & $\begin{array}{l}\text { (electr }{ }^{\star} \text { adj4 }\left(\text { record }^{*} \text { or medical }{ }^{*} \text { or }\right. \\
\left.\left.\text { health}^{\star} \text { or } \text { portal }^{*} \text { or } \text { tech }^{\star}\right)\right) \cdot \mathrm{mp} \text {. }\end{array}$ & 145224 \\
\hline 12 & cmc.mp. & 7877 \\
\hline 13 & $\begin{array}{l}\text { (telemed }^{*} \text { or tele-consult* or tele-med }{ }^{\star} \text { or } \\
\text { teleconsult* or telecom* or tele-com }{ }^{\star} \text { or } \\
\text { telehealth or tele-health).mp. }\end{array}$ & 29900 \\
\hline 14 & $\begin{array}{l}\left(\left(\text { virtual }^{\star} \text { or remote }\right.\right. \\
\text { consult }^{\star} \text { adj4 } \text { or }^{\text {visit }} \text { meet }^{\star} \text { or appoint } \\
\text { communicat }\end{array}$ & 5677 \\
\hline 15 & $\begin{array}{l}1 \text { or } 2 \text { or } 3 \text { or } 4 \text { or } 5 \text { or } 6 \text { or } 7 \text { or } 8 \text { or } 9 \text { or } \\
10 \text { or } 11 \text { or } 12 \text { or } 13 \text { or } 14\end{array}$ & 1327598 \\
\hline 16 & emotional intelligence/or empathy/ & 17802 \\
\hline 17 & $\begin{array}{l}\text { (empath* or compassion* or sympath*). } \\
\text { mp. }\end{array}$ & 155565 \\
\hline
\end{tabular}

$18 \quad\left(\right.$ (emotion* or suffer $^{\star}$ or illness* ${ }^{*}$ or unwell ${ }^{\star} \quad 29584$ or pain* or discomfort ${ }^{\star}$ or disabilit* or disabl $^{\star}$ ) adj4 (intelligen* or understand ${ }^{\star}$ or acknowledg* $^{\star}$ or consider ${ }^{\star}$ or recogniz ${ }^{\star}$ or recognition)).mp.

\begin{tabular}{|c|c|c|}
\hline 19 & $\begin{array}{l}\text { (humanis* or humanit* or humane). } \\
\mathrm{mp} \text {. }\end{array}$ & 19648 \\
\hline 20 & (dignity or dignif*).mp. & 6538 \\
\hline 21 & 16 or 17 or 18 or 19 or 20 & 207991 \\
\hline 22 & 15 and 21 & 9195 \\
\hline 23 & exp Communication/ & 272882 \\
\hline 24 & exp Professional-Patient Relations/ & 133459 \\
\hline 25 & communicat $^{\star} . \mathrm{mp}$. & 358275 \\
\hline 26 & $\begin{array}{l}\text { (interact* adj4 } \text { (patient }^{\star} \text { or client* or famil* } \\
\text { or person* or professional or user*)).mp. }\end{array}$ & 29221 \\
\hline 27 & $\begin{array}{l}\text { (relation* adj4 }^{*} \text { (patient* or client* or famil* } \\
\text { or person }{ }^{*} \text { or professional or user*)).mp. }\end{array}$ & 220054 \\
\hline 28 & interpersonal.mp. & 89099 \\
\hline 29 & (therap* adj2 alliance*).mp. & 2324 \\
\hline 30 & $\begin{array}{l}\text { ((person or patient }{ }^{\star} \text { or people or human*) } \\
\left.\left.\text { adj4 (centr }{ }^{\star} \text { or center }{ }^{\star}\right)\right) . \mathrm{mp} \text {. }\end{array}$ & 97925 \\
\hline 31 & $\begin{array}{l}23 \text { or } 24 \text { or } 25 \text { or } 26 \text { or } 27 \text { or } 28 \text { or } 29 \text { or } \\
30\end{array}$ & 864516 \\
\hline 32 & 22 and 31 & 1679 \\
\hline
\end{tabular}

Continued
Table 1 Continued

\begin{tabular}{llr}
\hline$\#$ & Searches & Results \\
\hline 33 & $\begin{array}{l}((\text { artificial* or remote or virtual or tele* }) \\
\text { adj4 (compassion* or empath* or }\end{array}$ & 331 \\
& $\begin{array}{l}\left.\left.\text { sympath }{ }^{*} \text { or emotion }\right)\right) . m p . \\
\text { 32 or 33 }\end{array}$ & 1998
\end{tabular}

'exp ' indicates that a subject heading is 'exploded' to include all of the narrower subject headings beneath it in the hierarchy.

' ' indicates that a term is a subject heading.

'?' holds the place of zero or one character.

'.mp.' indicates that a keyword should be searched in a collection of fields including title, abstract, author assigned keywords, subject headings, etc.

'Adj4' instructs the database to search for the words before and after it within 4 words of each other in either direction (adj2 searches within 2 words of each other).

where de-duplication will be performed. This EndNote library will then be exported as a spreadsheet to use as the foundation for the screening form. Any additional results from the handcomb process will be added to EndNote and documented in a separate spreadsheet.

\section{Study selection}

Two reviewers ( $\mathrm{RC}$ and $\mathrm{HC}$ or $\mathrm{RC}$ and $\mathrm{LS}$ ) will independently determine the eligibility of articles using a two-stage screening process consisting of a title and abstract scan followed by a full-text review. At the end of each stage, the findings will be compared and resolved by the reviewers or a third reviewer/senior investigator (AC, GS and/or DW) when consensus is not achieved. To be relevant for full-text review, the title and abstract must:

1. Discuss use of digital technology in healthcare;

2. Discuss aspects of caring, compassionate or empathetic communication (verbal or non-verbal); and/or

3. Include patient-provider relationship (ie, therapeutic alliance, person-centred or patient-centred care/ communication).

This review is inclusive of all literature published in English that is either a formative evaluation, regardless of methodology (ie, quantitative, qualitative or mixed methods, including usability studies or needs assessments), a case study or dissertation/theses. Dissertation/theses will be included to account for the maturity of literature on digital health innovations. Commentaries, editorials, letters and conference abstracts will be excluded. Additionally, seeing as our primary aim is to explore compassion towards others in clinical care, articles that primarily focused on self-compassion (eg, mindfulness-based stress reduction, compassion-focused psychotherapy) will be excluded.

Inter-rater reliability (IRR) will be established through the pilot where $20 \%$ of the Medline citations will be independently reviewed by three reviewers. From there, the IRR will be calculated and if there is an average Cohen's $\mathrm{K}$ of 0.70 , indicating substantial agreement, ${ }^{46}$ the remaining articles will be divided into two sets of 
pairs for independent review. This way, we ensure that all reviewers cultivate a common understanding of the inclusion criteria. If the threshold is not met, then the pilot will be run again. In either instance, when comparing results, reviewers will discuss and troubleshoot any challenges experienced during the title and abstract scan.

\section{Data collection}

A standardised charting form developed by the study team will be used to extract data. The domains include article details, study details, digital technology details and compassion details. Subdomains are outlined in table 2.

Prior to data extraction, this form will be piloted with three reviewers independently charting five articles, followed by validation by the senior investigators (AC, GS, DW). We will perform an iterative process where the form will be revised as needed, even during the data extraction if reviewers note and share any emerging themes not initially identified. A quality assessment will not be conducted as it is outside the scope of this review. ${ }^{32}$

\section{Data analysis}

A narrative synthesis approach will be taken to collate, summarise and report. ${ }^{1}$ This will include numerical summaries, using descriptive statistics, to report each high-level domain (article, study, digital technology and compassion details) ${ }^{41}$ in answering the first research question. Articles focused on the same digital technology will be grouped and treated as one unit. Subdomains in the digital technology and compassion details sections will be thematically analysed using inductive coding by three reviewers (RC, HC, LS), independently, to identify emerging themes. ${ }^{47}$ The first 5 or 10 articles will be reviewed (depending on the number of included articles) and coded independently, after which the reviewers will discuss and formulate a coding structure. This process will be repeated so that the coding structure becomes iteratively developed as more articles are reviewed. Once all articles have been reviewed, discussed and coded, the coding structure and findings will be presented for review by the senior members of the research team (AC, GS, DW). Specifically, for the first research question, thematic analysis will be used to identify commonalities within each defined component of compassion. For the second research question, thematic analysis of the compassion details will be twofold. First, the impact of technology (barriers/hindrances and facilitators/benefits) will be categorised into the defined components of compassion as outlined by the Oxford Handbook of Compassion Science. ${ }^{13}$ Any other components that surface will be noted. Second, data extracted from this section will be stratified according to the Framework for Compassionate Collaborative Care ${ }^{48}$ which follows the Donabedian Model $^{49}$ (ie, structures, processes and outcomes). Outcomes will be stratified by the level of impact at the patient, provider or systemic level. For the final research question, thematic analysis will be conducted on the reported professional practice needs, as well as noting
Table 2 Data charting domains and subdomains

\begin{tabular}{|c|c|}
\hline Domain & Subdomain \\
\hline \multirow[t]{3}{*}{ Article details } & Article type \\
\hline & Year \\
\hline & Country \\
\hline \multirow[t]{5}{*}{ Study details } & Study design \\
\hline & $\begin{array}{l}\text { Intervention } \\
\text { (ie, educational activity, clinical intervention, } \\
\text { simulation, etc) }\end{array}$ \\
\hline & Participants \\
\hline & Comparator (if any) \\
\hline & Outcomes \\
\hline
\end{tabular}

\begin{tabular}{|c|c|}
\hline \multirow{5}{*}{$\begin{array}{l}\text { Digital technology } \\
\text { details }\end{array}$} & Name of technology (if available) \\
\hline & $\begin{array}{l}\text { Type of technology (distinguished by primary } \\
\text { purpose) } \\
\text { Telemedicine and telehealth } \\
\text { Digitised medical devices } \\
\text { Electronic patient records } \\
\text { Prescribing and ordering } \\
\text { Clinical decision support } \\
\text { Digital communication } \\
\text { Digital health games }\end{array}$ \\
\hline & $\begin{array}{l}\text { Healthcare setting where technology was } \\
\text { used } \\
\text { (ie, mental health, primary care, surgical care, } \\
\text { etc) }\end{array}$ \\
\hline & $\begin{array}{l}\text { Specific tasks accomplished through } \\
\text { technology (eg, emailing laboratory results, } \\
\text { tracking mood) }\end{array}$ \\
\hline & $\begin{array}{l}\text { Relation to verbal or non-verbal } \\
\text { communication }\end{array}$ \\
\hline \multirow[t]{5}{*}{ Compassion details } & $\begin{array}{l}\text { Reported compassionate response as } \\
\text { defined by Post et al }(2014)^{12} \dagger \\
\text { 1. Initial recognition of suffering by an } \\
\text { individual } \\
\text { 2. Emotional response to this suffering } \\
\text { 3. Operative response to this suffering }\end{array}$ \\
\hline & $\begin{array}{l}\text { Reported barriers/hindrances of technology } \\
\text { on compassionate responseł }\end{array}$ \\
\hline & $\begin{array}{l}\text { Reported facilitators/benefits of technology } \\
\text { on compassionate response } \neq\end{array}$ \\
\hline & $\begin{array}{l}\text { Impact at which level: patient, provider and/ } \\
\text { or systemic } \neq\end{array}$ \\
\hline & $\begin{array}{l}\text { Reported professional practice needs (ie, } \\
\text { education/training)§ }\end{array}$ \\
\hline
\end{tabular}

*Modified from Lupton (2014) ${ }^{50}$ to focus on types of technologies used in patient-provider communication.

†Mapped to research question 1.

¥Mapped to research question 2.

§Mapped to research question 3.

any commonalities in theoretical constructs listed. To support this iterative process, three senior members of the research team (AC, GS, DW) as well as patients and AMS Phoenix advisory committee members will be engaged to audit decision-making, validate emerging themes and provide feedback on the results. We will retain an audit trail of each reviewer's independent coding, team meeting notes and all variations of the coding structure. 


\section{Patient and public involvement}

In addition to the senior members of the research team, patient partners and AMS Phoenix advisory committee members will be engaged to validate emerging themes and provide feedback on the results (particularly any themes that pertain to the patient-provider perspective). We will also invite these groups to contribute to the dissemination plan. Patients from the University Health Network's 'Patient Partners Program' will be invited to participate in this research process. This programme falls within the network's Patient Experience portfolio, in which they are committed to facilitating patient and caregiver contributions to important hospital planning and decision-making activities in order to provide truly compassionate, collaborative and responsive care (https://www.uhn.ca/corporate/AboutUHN/Patient_Experience/Pages/patient_ partners.aspx). The AMS Phoenix advisory committee for the project 'A Call to Caring' consists of an interdisciplinary group of healthcare professionals who advise strategic investment to promote and sustain education and practice environments that support the balance between human compassion and technical expertise (https:// www.ams-inc.on.ca/). By engaging these two groups, we will be following scoping review best-practices in which consumer and stakeholder involvement is encouraged to provide additional value to the study. ${ }^{41}$

\section{ETHICS AND DISSEMINATION}

This review presents the transparent and rigorous protocol that will be followed to inform and define 'digital compassion'. Research ethics approval and consent to participate is not required for this scoping review. By synthesising the digital literature on how compassion is discussed, we will advance knowledge on compassionate care in our everevolving healthcare setting. This way, we may determine what the current needs are for successful compassionate professional practice in order to adapt practice and related training guidelines to include consideration of digital technologies. This knowledge synthesis is a critical first step to providing evidence-based guidance on how digital health is to be incorporated into the practice and teaching of compassionate care. These results will inform resource development and strategy for the Associated Medical Services, a Canadian Charitable Organisation at the forefront of advancing research and leadership development in health and humanities, as part of their AMS Phoenix Project: A Call to Caring. These results will also be disseminated at relevant national and international conferences, such as the eHealth, Canada's National Conference and Tradeshow, American Medical Informatics Association (AMIA) and published in a peer-reviewed journal. We will leverage the expertise from this interdisciplinary study team to speak to the diversity of the health professions and their usage of digital health technologies as well as interdisciplinary dissemination of findings in medical education, nursing, psychiatry, psychology and informatics.
Acknowledgements We would like to thank the AMS Phoenix Project for their funding and leadership in supporting this work. We would like to acknowledge the support of the Medical Psychiatry Alliance, a collaborative health partnership of the University of Toronto, the Centre for Addiction and Mental Health, the Hospital for Sick Children, Trillium Health Partners, the Ontario Ministry of Health and Long-Term Care and an anonymous donor.

Contributors DW led the conceptualisation, design and development of this study and protocol. RC, HC and LS drafted the protocol and helped develop the research questions. MA, an experienced medical librarian, developed the search strategy, conducted the search and copy-edited the manuscript. TT, an experienced medical librarian, copy-edited the manuscript. AC and GS provided guidance to the study conceptualisation and design, and have revised all drafts of this manuscript for content and clarity. They will also assist with the validation of the emerging themes identified in the data analysis. All authors give approval to the publishing of this protocol manuscript.

Funding This work is supported by the Associated Medical Services, a Canadian charitable organisation at the forefront of advancing research and leadership development in health and humanities, as part of their AMS Phoenix Project: A Call to Caring.

\section{Competing interests None declared.}

Patient consent for publication Not required.

Provenance and peer review Not commissioned; externally peer reviewed.

Open access This is an open access article distributed in accordance with the Creative Commons Attribution Non Commercial (CC BY-NC 4.0) license, which permits others to distribute, remix, adapt, build upon this work non-commercially, and license their derivative works on different terms, provided the original work is properly cited, appropriate credit is given, any changes made indicated, and the use is non-commercial. See: http://creativecommons.org/licenses/by-nc/4.0/.

\section{REFERENCES}

1. Sinclair S, Norris JM, McConnell SJ, et al. Compassion: a scoping review of the healthcare literature. BMC Palliat Care 2016;15:6.

2. Davidson L. Recovery from psychosis: what's love got to do with it? Psychosis 2011;3:105-14.

3. Cooper J. Paul Brodwin: everyday ethics: voices from the front line of community psychiatry. Cult Med Psychiatry 2014;38:160-2.

4. Clayton AR. How 'person-centered' care helped guide me toward recovery from mental illness. Health Aff 2013;32:622-6.

5. Deegan PE. Human sexuality and mental illness: consumer viewpoints and recovery principles. Buckley P, Sexuality and serious mental illness. Amsterdam: Harwood Academic, 1999:21-33. edn.

6. Englander R, Cameron T, Ballard AJ, et al. Toward a common taxonomy of competency domains for the health professions and competencies for physicians. Acad Med 2013;88:1088-94.

7. Milestones. Accreditation Council for Graduate Medical Education. 2018 http://www.acgme.org/What-We-Do/Accreditation/Milestones/ Overview (accessed 7 Feb 2018).

8. Royal College of Physicians and Surgeons of Canada. CanMEDS: better standards, better physicians, better care. 2018 http://www. royalcollege.ca/rcsite/canmeds/canmeds-framework-e (accessed 7 Feb 2018).

9. Srinivasan M, Li ST, Meyers FJ, et al. "Teaching as a competency": competencies for medical educators. Acad Med 2011;86:1211-20.

10. Radey M, Figley CR. The social psychology of compassion. Clin Soc Work J 2007;35:207-14.

11. Swanson KM. Nursing as informed caring for the well-being of others. Image J Nurs Sch 1993;25:352-7.

12. Post SG, Ng LE, Fischel JE, et al. Routine, empathic and compassionate patient care: definitions, development, obstacles, education and beneficiaries. J Eval Clin Pract 2014;20:872-80.

13. Seppälä EM, Simon-Thomas E, Brown SL, et al. The Oxford handbook of compassion science. edn. New York, NY: Oxford University Press, 2017.

14. Canadian Medical Association. Health care transformation in Canada. 2011 http://policybase.cma.ca/dbtw-wpd/PolicyPDF/PD1005.PDF (accessed 7 Feb 2018).

15. Saks ER. The center cannot hold: my journey through madness. New York, NY: Hyperion Books, 2008:1-340.

16. Lee JL, Choudhry NK, Wu AW, et al. Patient use of email, Facebook, and physician websites to communicate with physicians: a national online survey of retail pharmacy users. J Gen Intern Med 2016;31:45-51. 
17. Oh H, Rizo C, Enkin M, et al. What is eHealth (3): a systematic review of published definitions. J Med Internet Res 2005;7:e1.

18. Eysenbach G, Jadad AR. Evidence-based patient choice and consumer health informatics in the Internet age. J Med Internet Res 2001;3:e19.

19. Canada Health Infoway. What is digital health? 2018 https://www. infoway-inforoute.ca/en/what-we-do/digital-health-and-you/what-isdigital-health (accessed $1 \mathrm{Feb}$ 2018).

20. Jones SS, Rudin RS, Perry T, et al. Health information technology: an updated systematic review with a focus on meaningful use. Ann Intern Med 2014;160:48-54.

21. Suggs LS. A 10-year retrospective of research in new technologies for health communication. $J$ Health Commun 2006;11:61-74.

22. Preece J. Empathic communities: balancing emotional and factual communication. Interact Comput 1999;12:63-77.

23. Moffatt JJ, Eley DS. The reported benefits of telehealth for rural Australians. Aust Health Rev 2010;34:276-81.

24. Swinglehurst $D$, Roberts $C$, Greenhalgh T. Opening up the 'black box' of the electronic patient record: a linguistic ethnographic study in general practice. Commun Med 2011;8:3-15.

25. Robinson K. Technology can't replace compassion in health care. Nurs Manage 2003;34:1

26. Lupton $\mathrm{D}$. The digitally engaged patient: Self-monitoring and self-care in the digital health era. Social Theory \& Health 2013;11:256-70.

27. Antoun J. Electronic mail communication between physicians and patients: a review of challenges and opportunities. Fam Pract 2016;33:121-6.

28. Seyffert M, Lagisetty $\mathrm{P}$, Landgraf $\mathrm{J}$, et al. Internet-delivered cognitive behavioral therapy to treat insomnia: a systematic review and metaanalysis. PLoS One 2016;11:e0149139.

29. Stiles-Shields C, Kwasny MJ, Cai X, et al. Therapeutic alliance in face-to-face and telephone-administered cognitive behavioral therapy. J Consult Clin Psychol 2014;82:349-54.

30. Niazkhani Z, Pirnejad H, Berg M, et al. The impact of computerized provider order entry systems on inpatient clinical workflow: a literature review. J Am Med Inform Assoc 2009;16:539-49.

31. Luxton DD, McCann RA, Bush NE, et al. mHealth for mental health: integrating smartphone technology in behavioral healthcare. Prof Psychol 2011;42:505-12.

32. Gagnon MP, Ngangue P, Payne-Gagnon J, et al. m-Health adoption by healthcare professionals: a systematic review. J Am Med Inform Assoc 2016;23:212-20.

33. Terry C, Cain J. The emerging issue of digital empathy. Am J Pharm Educ 2016;80:58.
34. Swinglehurst D. Displays of authority in the clinical consultation: a linguistic ethnographic study of the electronic patient record. Soc Sci Med 2014;118:17-26.

35. Associated Medical Services. Transformational trends in healthcare. 2017 http://www.ams-inc.on.ca/wp-content/uploads/2015/12/AMSTransformational-Trends-in-Healthcare.pdf (accessed 7 Feb 2018).

36. Johnston SC. Anticipating and training the physician of the future: the importance of caring in an age of artificial intelligence. Acad Med 2018;93:1105-1106.

37. Crawford A, Sunderii N, López J, et al. Defining competencies for the practice of telepsychiatry through an assessment of resident learning needs. BMC Med Educ 2016;16:28

38. Hilty DM, Crawford A, Teshima J, et al. A framework for telepsychiatric training and e-health: competency-based education, evaluation and implications. Int Rev Psychiatry 2015;27:569-92.

39. Ellaway RH, Coral J, Topps D, et al. Exploring digital professionalism Med Teach 2015;37:844-9.

40. Associated Medical Services. About AMS. 2018 http://www.ams-inc. on.ca/ (accessed 16 Feb 2018).

41. Arksey H, O'Malley L. Scoping studies: towards a methodological framework. Int J Soc Res Methodol 2005;8:19-32.

42. Colquhoun $\mathrm{H}$. Current best practices for the conduct of scoping reviews. EQUATOR Netw 2016. http://www. equator- network. org/wp- content/ uploads/ 2016/ 06/ Gerstein- Library- scopingreviews_May-12. pdf (accessed 1 February 2017).

43. Peters MD, Godfrey CM, Khalil H, et al. Guidance for conducting systematic scoping reviews. Int J Evid Based Healthc 2015;13:141-6.

44. Shamseer L, Moher D, Clarke M, et al. Preferred reporting items for systematic review and meta-analysis protocols (PRISMA-P) 2015: elaboration and explanation. BMJ 2015;349:97647.

45. Tricco AC, Lillie E, Zarin W, et al. PRISMA extension for scoping reviews (PRISMA-ScR): checklist and explanation. Ann Intern Med 2018;169:467-73.

46. Landis JR, Koch GG. The measurement of observer agreement for categorical data. Biometrics 1977;33:159-74.

47. Braun V, Clarke V. Using thematic analysis in psychology. Qual Res Psychol 2006;3:77-101.

48. Pfaff K, Markaki A. Compassionate collaborative care: an integrative review of quality indicators in end-of-life care. BMC Palliat Care 2017;16:65

49. Moore L, Lavoie A, Bourgeois G, et al. Donabedian's structureprocess-outcome quality of care model: validation in an integrated trauma system. J Trauma Acute Care Surg 2015;78:1168-75.

50. Lupton D. Critical perspectives on digital health technologies. Sociol Compass 2014:8:1344-59. 